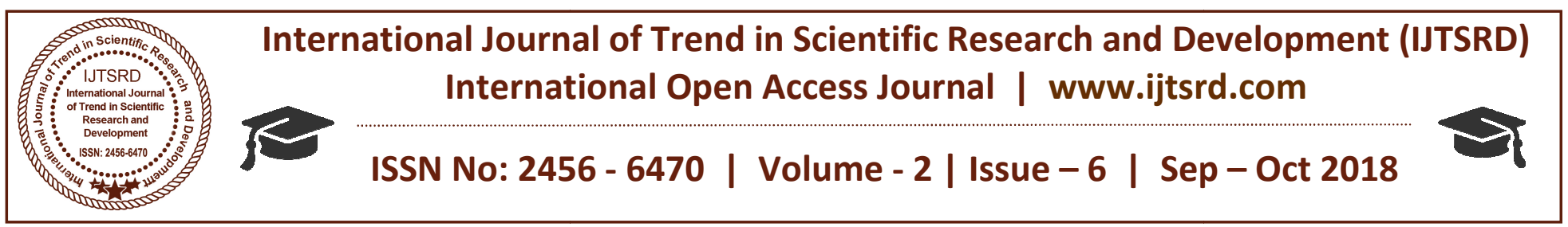

\title{
Multiple Pyaemic Abscess-An Unusual Presentation in Neonate: Case Report
}

\author{
Syeda Nishat Fathima \\ Assistant Professor, Department of Pharmacology, \\ Jayamukhi College of Pharmacy, Narsampet, Warangal, Telangana, India
}

\begin{abstract}
Pyaemia is a type of blood infection that leads to widespread abscesses of a metastatic nature. It is usually caused by the staphylococcus bacteria or by pus-forming organisms in the blood. It is a lifethreatening condition that ascends when the body's response to infection caused by bacteria causes injury to its own tissues and organs. Early recognition and focused management may improve the outcomes in this case. Apart from the timely administration of fluids and antibiotics, the management of Pyaemia also involves surgical Incision and drainage of infected fluid collections.
\end{abstract}

Keyword: Pyaemia, surgical Incision and drainage

\section{INTRODUCTION}

Sir William Osler defined pyaemia (or pyemia) as "a general disease, characterized by recurring chills and intermittent fever and the formation of abscesses in various parts, all of which result from the contamination of the blood by products arising from a focus contaminated by the bacteria of suppuration"1 Pyaemia is a type of septicemia that leads to widespread abscesses of a metastatic nature. It is usually caused by the pyogenic micro-organisms such as staphylococcus bacteria in the blood. The pus forming organism in the blood, forms abscess which is accompanied with the formation of multiple secondary metastasis abscesses in various parts of the body, which results from the invasion of microorganisms and their growth into colonies and in such a manner establishing independent centers of suppuration $^{2}$.

\section{CASE REPORT:}

A female Neonate of 19 days was presented with fever, painful left shoulder movement, mild erythema and swelling at the region of left shoulder join and restricted movement. She was unable to move the left hand and was referred to Orthopedic Surgeon. X-Ray of the neonate showed altered Echo texture of proximal Humerus-Osteomyelitis. Erb-Duchenne palsy has been ruled out as no neuro abnormality was detected. Next day baby was operated and the pus filled abscess was aspirated. The pus smear presented the presence of coagulase positive staphylococci grown in culture and Antimicrobial susceptibility test showed that the smear was resistant to ciprofloxin. Cefotaxime Injection 175 twice for one week and Injection of Amikacin 25gm Intravenous $12 \mathrm{hr}$ for two days was prescribed. Within three days of aspiration neonate developed abscess over right leg ankle and on the chest wall. 5-7 cc of Pus from right leg and 10-15 cc pus from Sternal region abscess were drained. an abscess cavity is cleaned and then packed with betadine (povidone-iodine) and hydrogen peroxide Neonate was immediately admitted in Pediatric Intensive Care Unit and prompt antibiotic therapy was started. The hematological Report of the Neonate during the course of treatment is presented in table 1 .

Treatment pattern included extended spectrum antibioticsVancomycin with Piperacillin-Tazobactam by Intravenous route as empirical therapy. The patient was discharged home on antibiotic prophylaxis with Linezolid syrup and Cefpodoxime syrup along withProwel sachet containing probiotic for seven days. Neonate completely recovered after 15 days of follow up and showed the normal hematological parameters with negative $\mathrm{C}$ - reactive protein. 
International Journal of Trend in Scientific Research and Development (IJTSRD) ISSN: 2456-6470

\begin{tabular}{|c|c|c|c|c|c|c|}
\hline \multirow{2}{*}{ S. No } & \multirow{2}{*}{ Hematological Parameters } & \multicolumn{5}{|c|}{ Age of Neonate } \\
\hline & & 19 Days & 22 Days & 25 Days & 25 Days & 32 Days \\
\hline 1. & Total WBC Count (cells/ cmm) & 7,200 & 27,700 & 20,100 & 16,700 & 11,200 \\
\hline 2. & Heamoglobin $(\mathrm{gm} \%)$ & 10.0 & 9.8 & 10.1 & 10.1 & 9 \\
\hline 3. & Neutrophils (\%) & 52 & 80 & 56 & 68 & 42 \\
\hline 4. & Eosinophils (\%) & 02 & 03 & 04 & 02 & $3 \%$ \\
\hline 5. & Basophils (\%) & 40 & 00 & 00 & 00 & $00 \%$ \\
\hline 6. & Lymphocytes (\%) & 00 & 15 & 35 & 25 & $50 \%$ \\
\hline 7. & Monocytes $(\%)$ & 06 & 02 & 05 & 05 & $05 \%$ \\
\hline 8. & Total RBC Count (million cells/ cmm) & 3.3 & 3.2 & 3. & 3.4 & 3.1 \\
\hline 9. & Haemocritvol \% & & 29 & 32 & 31 & 27 \\
\hline 10. & $\mathrm{MCV}(\mathrm{cmm})$ & & 92 & 93 & 91 & 88 \\
\hline 11. & $\mathrm{MCH} \longrightarrow$ & & 28 & 29 & 29 & 29 \\
\hline 12. & $\operatorname{MCHC}(\%)$ & SIEnth & 32 & 31 & 32 & $32 \%$ \\
\hline 13. & Platelet Count (Lakh cells/ cmm) & 2.2 & 6.0 & 7.2 & 6.4 & 4.7 \\
\hline 14. & ESR (MM at the end of first hour) & 8 & 70 & 46 & 26 & 32 \\
\hline 15. & Blood for CRP Test $(\mathrm{mg} / \mathrm{ml})$ & 07 & 77 & 31 & 25 & 06 \\
\hline 16. & Random Blood Glucose (mg/dl) & 82 & 96 & 60 & 83 & 84 \\
\hline
\end{tabular}

\section{Discussion:}

Pyemia is a condition in which metastatic abscesses arise and is characterized by fever of an intermittent type and by recurring chills. In case of pyaemia the pus passage into the blood of clots infected by microorganisms. Suppuration occurs and the clots become filled with the toxins of pyogenic bacteria $^{3}$.The abscess begins as a localized host acute inflammatory response to bacterial infection. Keratinocytes in addition to serving as a physical barrier to protect against microbes possess pattern recognition receptors that detect invading microbes and, in turn, signal the proinflammatory response as well as produce antimicrobial peptides that have direct activity against Staphylococcus organisms. As an abscess forms, it acquires several characteristic features such as center of the abscess i.e., focus contains an acute inflammatory exudate composed of many viable and necrotic neutrophils, tissue debris, fibrin, and live bacteria. Maturation of the abscess is followed by fibroblastic proliferation and tissue repair at the abscess margin and formation of a fibrous capsule at the periphery. Abscess formation is a mechanism used by the host to control and finally eradicate the pathogen ${ }^{4}$.

Incision and drainage is the recommended treatment for cutaneous abscesses. Despite lack of evidence, most experts recommend irrigation, breaking of loculations, and packing following incision and drainage ${ }^{5}$. Initial therapy for abscesses associated with severe and/or disseminated disease or those that fail to respond to incision and drainage is often empiric and relies on broad-spectrum antimicrobial agents ${ }^{6}$. The goals of antimicrobial therapy are to eradicate the contamination, reduce morbidity, and prevent complications. Knowledge of organisms and resistance patterns plays an vital role in appropriate antimicrobial selection. Oral antibiotic options include clindamycin, trimethoprim/sulfamethoxazole, a tetracycline (doxycycline or minocycline), and linezolid. For hospitalized patients with complicated major abscesses empiric therapy should be considered pending culture results, in addition to surgical debridement and broad-spectrum antibiotics. Empiric therapy options include intravenous vancomycin, linezolid (600 mg orally or intravenously twice per day), daptomycin (4 mg per kg intravenously once per day), telavancin (10 mg per kg intravenously once per day), or clindamycin (600 mg intravenously or orally three times per day). A beta-lactam antibiotic (e.g., cefazolin) may be considered ${ }^{7}$.Vancomycin with piperacillin-tazobactam is used as empirical therapy for critically ill patients ${ }^{8}$. The early diagnosis and adequate treatment of this life-threatening abscess may result in excellent prognosis. 


\section{References:}

1. Sir William Osler. The Principles and Practice of Medicine: Designed for the Use of Practitioners and Students of Medicine. Vol. 82., Appletons' medical library , 1892, 116, Chapter 2: Pyemia

2. Ferdinand J S G. Dental Medicine. A Manual of Dental Materia Medica and Therapeutics. Edn 7, P. Blakiston's Son \& Co, 1901, 184-185.

3. Da Costa, John Chalmers. Modern Surgery Chapter 10. Septicemia and PyemiaEdn 4, John Chalmers Da Costa, 1903, Paper 49.

4. Kobayashi S D, Malachowa N, De Leo F R. Pathogenesis of Staphylococcus aureus
Abscesses. The American Journal of Pathology. 2015; 185(6):1518-1527.

5. Korownyk C, Allan G M. Evidence-based approach to abscess management. Canadian Family Physician. 2007; 53(10):1680-1684.

6. Leekha S, Terrell C L, Edson R S. General Principles of Antimicrobial Therapy. Mayo Clinic Proceedings. 2011; 86(2):156-167.

7. Mara L. Practice Guidelines IDSA Guidelines on the Treatment of MRSA Infections in Adults and Children. American Family Physician Journal. 201; 84(4):455-463.

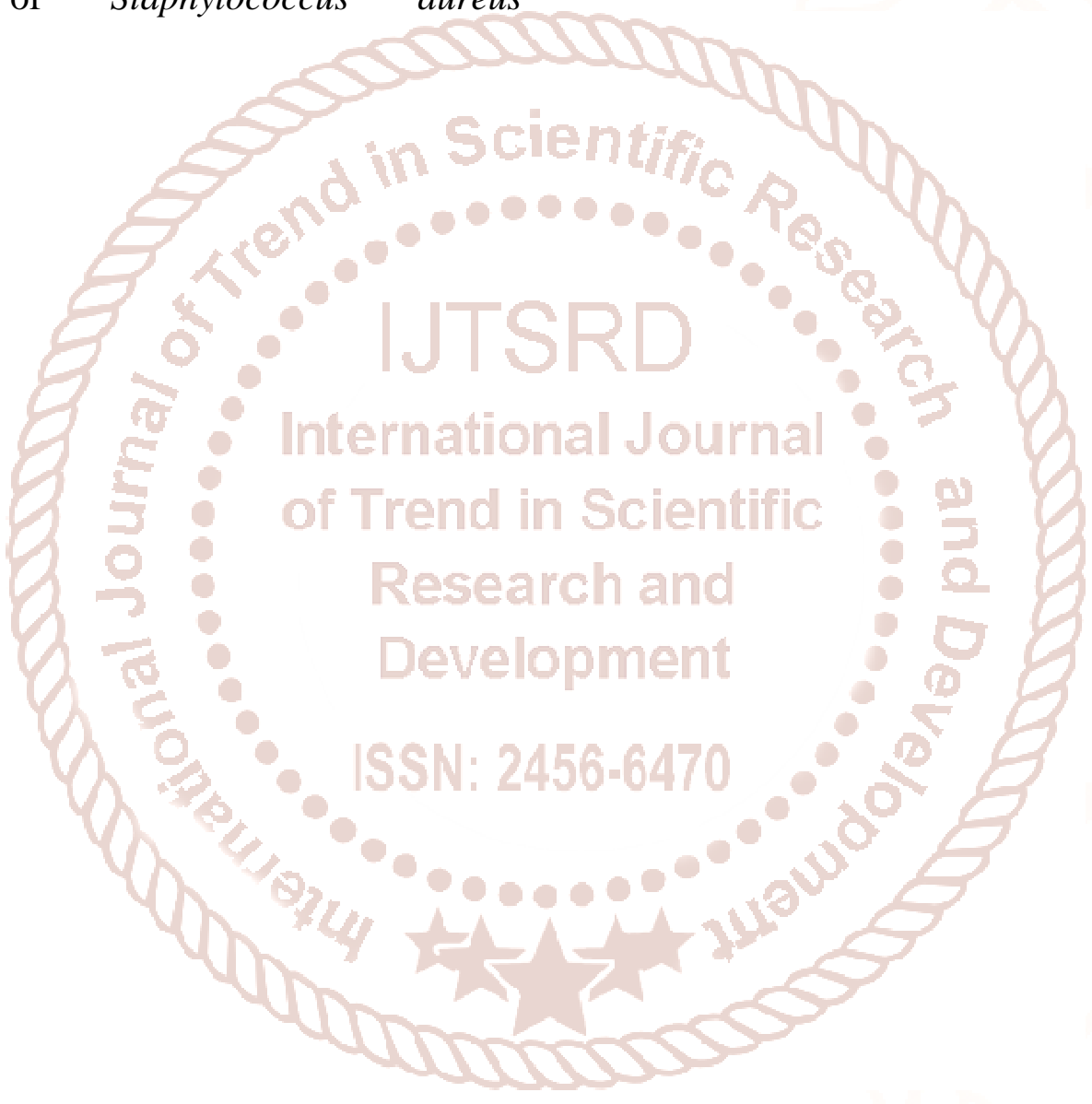

